\title{
1 Leptospira-rat-human relationship in Luzon, Philippines
}

2

3 Sharon Yvette Angelina M. Villanueva ${ }^{\mathrm{a},{ }^{*},}$, Mitsumasa Saito ${ }^{\mathrm{a}}$, Rubelia A. Baterna ${ }^{\mathrm{b}}$,

4 Crystal Amiel M. Estrada ${ }^{\text {b }}$, Ana Kriselda B. Riverab ${ }^{\text {b }}$ Micaella C. Dato ${ }^{\text {b }}$

5 Pia Regina Fatima C. Zamora ${ }^{c}$, Takaya Segawa ${ }^{a}$, Lolita L. Cavinta ${ }^{b}$, Takashi Fukui ,

6 Toshiyuki Masuzawa ${ }^{d}$, Yasutake Yanagihara ${ }^{c}$, Nina G. Gloriani ${ }^{b}$, Shin-ichi Yoshida ${ }^{a}$

7

$8{ }^{a}$ Department of Bacteriology, Faculty of Medical Sciences, Kyushu University, Fukuoka,

9 812-8582, Japan

$10{ }^{b}$ Department of Medical Microbiology, College of Public Health, University of the

11 Philippines-Manila, Manila, 1000, Philippines

$12{ }^{\mathrm{c} C}$ College of Medicine, University of the Philippines-Manila, Manila, 1000, Philippines

13

${ }^{\mathrm{d}}$ Department of Immunology and Microbiology, School of Pharmaceutical Sciences, Chiba

14 Institute of Science, Chiba, 288-0025, Japan

15

16 *Corresponding author. Tel: +81-92-642-6128; Fax: +81-92-642-6133.

17 E-mail address: svillan@bact.med.kyushu-u.ac.jp (S. Y. A. M. Villanueva) 


\section{Abstract}

20

21 Leptospirosis is a zoonotic infection that is caused by the pathogenic species of

22 Leptospira. Rats are the most important reservoirs of this zoonosis. Our study aimed to

23 characterize Leptospira isolates from humans and rats and elucidate the Leptospira-rat-human

24 relationship in Luzon, Philippines. Forty strains were isolated from humans and rats. The

25 isolates were confirmed to be Leptospira and pathogenic through $r r l-$ and flaB-PCR,

26 respectively. Around $73 \%$ of the isolates were found to be lethal to hamsters. Serotyping

27 showed that there were mainly three predominant leptospiral serogroups in the study areas

namely Pyrogenes, Bataviae, and Grippotyphosa. Gyrase B gene sequence analysis showed

29 that all the isolates belonged to L. interrogans. Most had 100\% similarity with serovar

30 Manilae (15/40), serovar Losbanos (8/40), and serogroup Grippotyphosa (8/40). Strains from

31 each group had highly identical pulsed-field gel electrophoresis patterns and were further

32 grouped as A (Pyrogenes, 14), B (Bataviae, 8), and C (Grippotyphosa, 10). Results further

33 revealed that similar serotypes were isolated from both humans and rats in the same areas. It

34 is suggested that these three predominant groups with highly similar intra-group PFGE

35 patterns may have been primarily transmitted by rats and persistently caused leptospirosis in 
36 humans particularly in the Luzon islands.

37

Keywords. Leptospira; rat; human; PFGE; flaB-PCR; 23S rDNA (rrl)-PCR; gyrB

39 sequence analysis; geographic information system (GIS); pathogenicity; hamster

40 
41

42

43

44

45

46

47

42

\section{Introduction}

Leptospirosis, a zoonosis common in tropical and sub-tropical countries, affects

millions of people annually [1]. Rodents, specifically rats, are the most important reservoirs

of this infection. They maintain Leptospira in their kidneys, continuously shed the organisms

in their urine, and contaminate the environment (i.e., water and soil). Humans and other

animals become infected, mainly through their skin and mucous membranes, when they come

in contact with a leptospire-contaminated environment [2].

The close relationship among Leptospira, rats, and humans has been described in

several studies [3-5]. However, there have been very few reports characterizing the

relationship between rat and human isolates using serologic or molecular epidemiologic

methods. It is probably due to difficulties in the simultaneous isolation of Leptospira from

rats and humans.

To perform epidemiologic studies on leptospirosis, serovar/serogroup identification of

isolates is essential. However, it is not routinely performed in most laboratories because there

are more than 250 pathogenic serovars organized into 24 serogroups based on antigenic

relatedness, and the maintenance of large panels of reference antisera and live antigens is 
required [6]. In order to overcome these problems, some molecular techniques discriminating

59 Leptospira isolates for epidemiological studies were developed. Most of them can only

60 identify until the species level (FAFLP, RFLP, 16S rRNA sequence analysis, etc.). MLST has

61 been reported to be useful for phylogenetic study [7]. Some researchers reported that

62 pulsed-field gel electrophoresis (PFGE) is an alternative method for the identification of

63 Leptospira serovars [7-9]. PFGE has the ability of differentiating between strains of the same

64 serovars that belong to different Leptospira species [10]. PFGE was often used to compare

65 Leptospira isolates in previously reported epidemiological studies. Methods often used in

66 identifying the species of Leptospira are DNA-DNA hybridization and 16S rRNA gene (rrs)

67 sequence analysis. However, there have been several reports that used the housekeeping

68 enzyme, DNA gyrase B subunit gene $(\operatorname{gyr} B)$, for identification of Leptospira isolates [11-13].

69 Slack et al [13] reported that the nucleotide divergence of gyrB was greater than 16S rRNA

$70 \quad(3.5-16.1 \%$ vs $0.1-1.4 \%)$.

71 In the Philippines, many people are infected with Leptospira. Outbreaks usually occur

72 during the rainy season (June to November) and just after the rainy season in flood-prone

73 areas [14-16], although there are unofficial reports of outbreaks occurring even during the dry

74 season especially in rural areas (e.g., areas with rice fields). We previously reported about the 
75 genetic and serological characterization of rat isolates in the Philippines [17]. The study was

76 carried out in two areas, Metro Manila and Laguna, and we isolated 50 Leptospira strains

77 from 46 wild rats. Based on gyrB sequencing and PFGE analysis, four groups of rat isolates

78 were found: L. interrogans serovar Manilae; serovar Losbanos; and serogroup Grippotyphosa;

79 and, L. borgpetersenii serogroup Javanica. Our previous study showed that there was a

80 difference in geographic distribution among Leptospira species and serovar/serogroup [17].

81 The association between rat isolates and human isolates were not analyzed in the said study.

82 However, those rat isolates caused hematuria, icterus, and pulmonary hemorrhage, which are

83 the symptoms of severe human leptospirosis, and led to the death of golden Syrian hamsters

84 [18]. From these results, it was expected that these two Leptospira serovars and two

85 serogroups are circulating among rats, and that the rats may be one of the possible

86 transmission sources of leptospirosis in the Philippines.

87 The present study was carried out in order to clarify the relation between rat and human

88 Leptospira isolates in the Philippines. We successfully obtained clinical isolates from

89 leptospirosis patients and new isolates from wild rats during the same period. Then, we

90 investigated the serotype, genotype, and geographic distribution of the isolates, and evaluated

91 the Leptospira-rat-human relationship in the Philippines, particularly in Luzon. Moreover, we 
92 compared the PFGE patterns and the gyrB sequences of the isolates in this study with those of

93 previous isolates from rats.

94 Knowledge on the Leptospira-rat-human relationship is very essential in

95 formulating countermeasures (e.g., vaccine) to combat leptospirosis.

96

\section{2. Materials and methods}

98

992.1 Isolation of leptospires

100

\subsubsection{Humans}

101 Blood and urine samples were obtained from suspected leptospirosis patients referred by physicians to the Department of Medical Microbiology, College of Public Health,

103 University of the Philippines Manila between 2010 and 2011. As previously reported [6, 17],

104 one to three drops of blood and urine were cultured in modified Korthof's medium

105 supplemented with $10 \%$ rabbit serum, kept at $30^{\circ} \mathrm{C}$ incubator, and checked weekly for leptospiral growth until 3 months. 
110 Manila (cities of Manila and Makati), and rice fields in Nueva Ecija and Iloilo. Wire-mesh

111 traps with baits were left overnight. The trapped rats were sacrificed by carbon dioxide

112 inhalation. Blood was collected through cardiac puncture and urine was aseptically collected

113 by bladder puncture with a needle. Rat kidneys were aseptically removed and emulsified with

114 a 2.5-ml syringe based on a previously reported method [17]. Urine in bladder or empty

115 urinary bladder, and kidneys were cultured in 5-fluorouracil-containing modified Korthof's

116 medium. These cultures were incubated overnight at $30^{\circ} \mathrm{C}$. For the bladder and kidney

117 cultures, $500 \mu \mathrm{l}$ of the supernatant was sub-cultured into $4 \mathrm{ml}$ of fresh medium the next day

118 and were kept at $30^{\circ} \mathrm{C}$. All the cultures were examined weekly for leptospiral growth for three

119 months. Species of rats were identified based on morphological features such as the color of

120 ventral and dorsal fur, tail length compared to the length of head and body, number of pairs of

121 mammae (females), and weight [19]. 
126 Illustra Bacterial GenomicPrep Mini Spin Kit (GE Healthcare, Buckinghamshire, UK)

127 following the protocol for Gram-negative bacteria.

128

$129 \quad 2.3$ Typing of isolates

130 2.3.1 23S rDNA (rrl)-polymerase chain reaction (PCR)

131 Woo et al. [20] reported that the 23S rDNA ( $r r l)$-PCR was capable of detecting all

132 Leptospira species. For 23S rDNA-PCR, the primers, rrl-F (5'-

133 GACCCGAAGCCTGTCGAG -3') and rrl-R (5'- GCCATGCTTAGTCCCGATTAC-3') were

134 used. Each PCR solution (50 $\mu$ l) consisted of $1 \times$ ExTaq Buffer (Takara, Otsu, Shiga, Japan),

$135100 \mu \mathrm{M}$ each dNTP, $0.25 \mu \mathrm{M}$ of each universal primer, $100 \mathrm{ng}$ of extracted DNA and 1.25

136 units of Ex Taq ${ }^{\text {TM }}$ HS DNA polymerase (Takara, Otsu, Shiga, Japan). Amplification was

137 carried out in a thermal cycler (Program Temp Control System PC-320, ASTEC Co. Ltd.,

138 Fukuoka, Japan) under the following conditions: 30 cycles of $94^{\circ} \mathrm{C}$ for $20 \mathrm{sec}, 54^{\circ} \mathrm{C}$ for 30

$139 \mathrm{sec}, 72^{\circ} \mathrm{C}$ for $1 \mathrm{~min}$ and a final extension at $72^{\circ} \mathrm{C}$ for $6 \mathrm{~min}$. PCR amplification was confirmed

140 by electrophoresis on $1.5 \%$ agarose gels.

141

142 2.3.2 Flagellin B gene (flaB)-PCR 
144 pathogenic Leptospira strains. For flaB-PCR, the primers, L-flaB-F1

145 (5'-CTCACCGTTCTCTAAAGTTCAAC-3') and L-flaB-R1

146 (5'-TGAATTCGGTTTCATATTTGCC-3') were used. The preparation of PCR solution, DNA

147 amplification, and electrophoresis were done in the same way as that for 23S rDNA PCR

148 described above. 2.3.3 Gyrase B gene (gyrB)-PCR and sequence analysis The DNA gyrase B subunit gene ( $g y r B)$ of all the Leptospira isolates from humans and rats was amplified using the primer set UP1TL (CAYGCNGGNGGNAARTTYGA) and UP2rTL (TCNACRTCNGCRTCNGTCAT) [22] under the following conditions: 30 cycles of

154 denaturing at $94^{\circ} \mathrm{C}$ for 30 seconds, annealing at $55^{\circ} \mathrm{C}$ for 60 seconds, and extension at $72^{\circ} \mathrm{C}$

155 for 90 seconds. Final extension is at $72^{\circ} \mathrm{C}$ for 10 minutes. Sequencing of gyrB was done using

156 the aforementioned primer set and LgyrF (GGTCTTTCCGGAGAAGATG) and LgyrR

157 (GAATTTGAATTTGAGGTTGAGG) [12]. Sequence analysis was done based on a previous

158 study [17]. Briefly, 3130 genetic analyzer (Applied Biosystems, Foster City, CA) was used to

159 analyze the sequences. The alignment of sequences, neighbor-joining phylogenetic tree 
160 construction, and bootstrap analysis were performed by using version 7.1 software package of

161 Lasergene (DNASTAR Madison, WI). All the gyrB sequences of the Leptospira isolates in

162 this study and 4 strains in the previous study (i.e., K64, K37, K6, and K5) [17] have been

163 deposited and assigned DNA Data Bank of Japan (DDBJ)/European Molecular Biology

164 Laboratory (EMBL)/GenBank accession numbers AB968314 to AB968358.

165

166 2.3.4 NotI enzyme digestion and pulsed-field gel electrophoresis analysis

167 Pulsed-field gel electrophoresis (PFGE) was carried out based on the method previously described [23]. The patterns of leptospiral DNA digested with NotI were analyzed

169 using GelCompar II software (Applied Maths, Inc., Austin, TX). Dendrograms were created

170 by UPGMA cluster analyses based on the Dice band-based coefficient. Band comparison

171 settings of $1.2 \%$ optimization and $1 \%$ position tolerance were used. The fingerprint patterns

172 of the isolates in this study were compared with that of previous isolates from the Philippines

173 such as strains LT 398 [24] and K64 [17] (serogroup Pyrogenes, serovar Manilae); strains LT

174 101-69 [24] and K37 [17] (serogroup Bataviae, serovar Losbanos); strains UP-BL-FR13

175 (Yanagihara Y, et al, unpublished data) and K5 [17] (serogroup Grippotyphosa); and, strain

176 K6 (serogroup Javanica serovar Javanica) [17]. 


\subsubsection{Serotyping}

through microscopic agglutination test (MAT) using monoclonal and polyclonal antibodies

181 raised against several Leptospira serovars [25]. Mouse monoclonal antibodies were raised

182 against 4 previously isolated leptospires from the Philippines [17] namely L. interrogans

183 serogroup Pyrogenes serovar Manilae (strain K64), serogroup Bataviae serovar Losbanos

184 (strain K42), serogroup Grippotyphosa (strain K5), and L. borgpetersenii serogroup Javanica

185 serovar Javanica (strain K6). The monoclonal antibodies were developed based on the method

186 by Masuzawa et al [26] using P3-X63-Ag8-U1 mouse myeloma cells. Rabbit polyclonal

187 antibodies, on the other hand, were raised against L. interrogans serovar Manilae (strains LT

188398 and K64), serovar Losbanos (strains LT 101-69 and K37), serovar Icterohaemorrhagiae

189 (strain Ictero No. 1), serovar Pyrogenes (strain Pyrogenes), serovar Canicola (strains Hond

190 Utrecht and Moulton), L. interrogans (strain UP-BL-FR13), L. borgpetersenii serovar Poi

191 (strain Poi), and L. biflexa serovar Patoc (strain Patoc 1) based on a method specified in the

192 WHO Guidance [6] with some modifications [18]. 
194

195

196

197

\subsection{Pathogenicity of isolates in golden Syrian hamsters}

The pathogenicity of 29 selected Leptospira isolates was tested using four-week-old male golden Syrian hamsters as previously reported with some modifications [17-18]. Briefly, hamsters were subcutaneously injected with $1 \mathrm{ml}$ of $10^{7}$ and $10^{5}$ of each strain and the animals were observed for 21 days. Moribund and surviving hamsters were sacrificed using sevoflurane (Maruishi Pharmaceutical, Japan).

\subsection{Geographic Information System (GIS)}

The addresses of the patients as well as the trapping sites of the rats were obtained and plotted on the map using the GIS software, ArcGIS.

\subsection{Human and Animal Ethics}

approved by the Ethical and Technical Review Boards and the Institutional Animal Care and

All experiments involving human samples and animals were reviewed and

Use Committee (IACUC) of the University of the Philippines Manila. For human samples, the patients or their guardian were given both verbal and written explanation of the study in 
210 English or the vernacular. After thorough explanation, informed consents were obtained, and

211 samples were collected.

212

213 3. Results

214

2153.1 Isolation and 23S rDNA PCR of leptospires from human patients and rats

216 In the present study carried out from 2010 to 2011, a total of 40 strains of

217 leptospires were obtained: 27 from humans and 13 from rats (Table 1). One out of 26 patients

218 had positive blood and urine cultures so the total number of human isolates obtained was 21

219 strains from blood and 6 strains from urine. Leptospires were isolated from the urine in the

220 bladder (5 isolates), empty urinary bladders ( 2 isolates), and kidneys (6 isolates) of 9 out of

22156 trapped rats. Four of these rats had positive kidney and urine cultures. Based on their

222 morphological characteristics [19], 4 of the 9 culture-positive rats were Rattus norvegicus, 2

223 were $R$. tanezumi, while the 3 were not identified due to missing information. All the 40

224 isolates from humans and rats were confirmed to be leptospires under the dark-field

225 microscope because they showed the characteristic thin helical structures with prominent 
226 hooked ends and motility. Furthermore, all the isolates were determined to be Leptospira

227 species by the presence of 482 base pair bands in 23S rDNA PCR (data not shown).

2293.2 flaB-PCR of the isolates

230 The flaB-PCR assay produced positive PCR products with a size of $793 \mathrm{bp}$ for all

23140 isolates from humans and rats (data not shown). These results suggested that all the

232 isolates were pathogenic.

2343.3 PFGE, gyrB sequence analysis, and identification of serogroups of Leptospira isolates

235 The NotI restriction patterns of the 40 Leptospira isolates in this study and the

236 seven strains previously isolated in the Philippines were compared by phylogenetic tree

237 analysis. As shown in Fig. 1, the band patterns of the 40 isolates were categorized into four

238 groups: A, B, C, and others. The number of isolates belonging to each group was 14 (35\%), 8

239 (20\%), $8(20 \%)$, and $10(25 \%)$, respectively. The isolates which belonged to Groups A, B or C

240 accounted for $75 \%$ of the total number of isolates in from 2010-2012, suggesting a high

241 frequency of isolation of leptospires belonging to these 3 groups. 
Gyrase B gene sequence analysis revealed that all the isolates belonged to $L$.

243 interrogans (Fig. 2). Strains with Group A PFGE pattern, except PI-17, had identical

244 sequences with strains LT 398 and K64 of serovar Manilae (38\%). Group B and C strains had

245 identical gyrB sequences with serovar Losbanos and serogroup Grippotyphosa, respectively

246 (both 20\%). It is interesting to note that strains belonging to Group C had identical sequences

247 to Group A except for one-base substitution. Strains belonging to the others group had similar

248 sequences with serovar Icterohaemorrhagiae (2 strains) and serovar Canicola (1 strain).

249 Strains PI-35 and 43 had sequence similar (99.9\%) to serovar Pyrogenes. There is 1 base

250 difference between these 2 strains and serovar Pyrogenes strain Salinem. Strains PI-14, 34, 40,

251 and 42 were similar to serovar Naam. However, BLAST search revealed that these 6 strains

252 had $100 \%$ identical gyrB sequences to different serovars belonging to different serogroups.

253 Therefore, these strains could not be identified until the serovar level (Fig. 2).

254 The serogroup of each isolate was identified by MAT (Table 2, Fig. 1, Fig. 2).

255 Together with the PFGE results in Fig. 1 and gyrB sequence results in Fig. 2, group A was

256 composed of strains belonging to serogroup Pyrogenes. Group B strains reacted with antisera

257 for serogroup Bataviae. And, all the isolates in Group C were identified as serogroup

258 Grippotyphosa. 
It is noteworthy that the 3 predominant groups contained both human and rat

260 isolates. In other words, human and rat isolates in each group had highly similar fingerprint

261 patterns. The PFGE patterns of Group A isolates were indistinguishable to that of strain K64

262 (serogroup Pyrogenes serovar Manilae), which we isolated from rats in 2006 and categorized

263 into 'group A' in our previous study [17]. Similarly, Group B and C strains showed the

264 indistinguishable PFGE patterns of strain K37 (serogroup Bataviae serovar Losbanos) and

265 strain K5 (serogroup Grippotyphosa) isolated from rats in 2006 and categorized into 'group

266 B' and 'group C' in our previous report [17], respectively (Fig. 1). Moreover, the fingerprint

267 pattern of Group A strains was also identical to that of strain LT 398 (serogroup Pyrogenes

268 serovar Manilae), which was a reference strain isolated from a rat in Manila in 1957 [24].

269 Likewise, Group B strains were found to be identical to strain LT 101-69 (serogroup Bataviae

270 serovar Losbanos) isolated from a rat before 2006 [24], and Group C strains were considered

271 to be indistinguishable to strain UP-BL-FR13 (serogroup Grippotyphosa) isolated from rats in

2722000 (Fig. 1).

273 The remaining ten isolates were grouped into three serogroups namely: Canicola,

274 Icterohaemorrhagiae, and non-group A Pyrogenes. Seven of these belonged to serogroup 
275 Pyrogenes and showed four PFGE patterns that were distinct from the fingerprints of strains

276 in Group A, which are also under the Pyrogenes group.

278 Manilae and serogroup Grippotyphosa. Interestingly, 1 R. norvegicus harbored both Manilae

279 and Losbanos in its kidney and urine, respectively. Our results showed that no particular rat

280 species harbored specific serovar or serogroup.

282 3.4 Pathogenicity of the isolates in golden Syrian hamsters

To examine the pathogenicity of 29 selected isolates, hamsters were inoculated

284 subcutaneously with $10^{5}$ or $10^{7}$ of each isolate and were observed for 21 days. Thirteen of 18

285 isolates (72\%) from humans and 8 of 11 from rats $(73 \%)$, regardless of serotype and dose,

286 were found to be lethal to hamsters. The animals infected with the isolates became moribund

287 from the $6^{\text {th }}$ to the $12^{\text {th }}$ day after infection. Sick hamsters were observed to have ruffled fur,

288 decreased food and water intake, continuous decrease in body weight until moribundity, and

289 decreased mobility with some having prostration.

290

291

3.5 Distribution of Leptospira in the study sites 
293 geographic information system (GIS). The patients' addresses as well as the areas where rats

294 were trapped were plotted on the map (Fig. 3) using ArcGIS software.

295 Rat and human isolates that belonged to Group A (serogroup Pyrogenes) were

296 found to be mainly isolated in Metro Manila (National Capital Region). It is noteworthy that

297 the strains in Group A isolated from 4 patients with leptospirosis were also found to be in the

298 same area where leptospires from the kidneys and/or urine of 8 rats were also isolated. Group

299 B strains (serogroup Bataviae) were found to be isolated only in Metro Manila and its

300 surrounding areas (i.e., Laguna and Cavite). In the City of Manila, both rat and human

301 isolates in Group B were obtained. In contrast, all strains of Group C (serogroup

302 Grippotyphosa) were isolated in Pangasinan and Nueva Ecija (Regions I and III, respectively),

303 both are located north of Metro Manila. The strains belonging to non-group A Pyrogenes

304 showed a wide geographic distribution. An important finding with regards to the distribution

305 of the strains is that those strains belonging to Group A had high fingerprint pattern similarity

306 whereas those strains belonging to non-group A Pyrogenes showed diverse PFGE patterns.

307 The two rat isolates that were obtained in Iloilo City of Panay Island, located in the Visayas,

308 were found to be indistinguishable by PFGE, and were identified, through MAT, as serogroup 
309 Icterohaemorrhagiae. Sequence analysis of the gyrB gene of these 2 isolates showed $100 \%$

310 identity to that of serovar Icterohaemorrhagiae.

312 4. Discussion

313

There are several previous reports on the dominant clone of Leptospira associated

315 with outbreaks of human leptospirosis based on partial $r r s$ and $\sec Y$ sequences [27], MLST

316 [28], arbitrarily primed PCR [29], and PFGE [7]. In this study, we analyzed 27 Leptospira

317 isolates from blood and urine of patients with leptospirosis, and 13 isolates from rats. We

318 found that all of these isolates belonged to L. interrogans based on gyrB sequence analysis

319 (Fig. 2). Results also revealed that there were three predominant groups: serovars Manilae and

320 Losbanos, and serogroup Grippotyphosa (Figs. 1 and 2). The isolates of each predominant

321 group showed highly identical PFGE patterns (Group A, B, and C in Fig. 1). The results of

322 PFGE and serotyping of the isolates were in congruence with the results of gyrB sequencing.

323 A very important finding in this study is that the isolates of each human leptospiral

324 group was also obtained from rats either in the same year or a year apart and some were in the

325 same areas. We also showed that the fingerprint patterns of each three Leptospira groups were 
326 identical with that of previous isolates from rats in 2006 [17]. Moreover, the fingerprint

327 patterns of reference strains isolated before 2006 (i.e., strains LT 398 and LT 101-69) were

328 also found to be similar with that of each of the three Leptospira groups. This is the first study

329 demonstrating that the three predominant Leptospira groups have been circulating among rats

330 during the past decades or more than half a century particularly in the biggest island of the

331 Philippines, Luzon.

332 Isolation results showed that one human leptospirosis patient had leptospires in his

333 blood and urine, and 4 rats had these organisms in their kidneys and urine. The leptospires

334 obtained from the blood and urine of the patient had $100 \%$ identical gyrB sequences and

335 similar band patterns, and were classified as belonging to Group B (serovar Losbanos). The

336 Leptospira isolates from the kidneys and urine of 3 of the 4 rats had similar band patterns to

337 Group A (serovar Manilae). However, one interesting finding in our study was that 1 of the 4

338 rats harbored different types of Leptospira in its kidney and urine. The isolate from the kidney

339 had $100 \%$ gyrB sequence identity and similar fingerprint patterns with Group A (serovar

340 Manilae) while that from the urine was identical to Group B (serovar Losbanos). We have

341 also reported this phenomenon in our previous study [17], wherein 4 rats harbored different

342 types of Leptospira (i.e., serovar Manilae and serogroup Javanica). Furthermore, results 
343 showed that serovar Manilae and serogroup Grippotyphosa were isolated from rats regardless

344 of their species (i.e., $R$. norvegicus and R. tanezumi).

345 Geoprocessing techniques such as geographic information system (GIS) are

346 important tools in health studies especially in determining the spatial distribution of certain

347 diseases [30]. In our study, GIS was used to plot the distribution of Leptospira isolates. It was

348 found that the Leptospira groups were unequally distributed in Philippines, to be specific,

349 Group A strains (except for one isolate, PI-44) were isolated in Metro Manila; Group B

350 strains were obtained in Metro Manila and its surrounding areas; and, Group C strains were

351 collected in Pangasinan and Nueva Ecija, located north of Metro Manila. Strains identical to

352 human isolates were always obtained from rats trapped in the same areas where leptospirosis

353 patients lived. It is assumed that leptospirosis is not usually caused by human-to-human

354 transmission, therefore, these three predominant Leptospira groups are considered to be

355 transmitted from rats to humans. These findings revealed that the dominant Leptospira strains

356 are maintained among the wild rats and occasionally transmitted to humans through the urine

357 of reservoir hosts (i.e., rats) or urine-contaminated environment. The reason why the three

358 dominant groups were selected in Luzon is unclear, however, these groups may have some

359 selective advantage, such as high bacterial load in the urine of rats, increased resistance to 
360 desiccation, and high ability to invade susceptible animals or humans.

361 It is interesting to know the details about the transmission of Leptospira organisms

362 in rat colonies. We hypothesize that there might be horizontal or vertical transmission among

363 the rats. Aside from these transmission routes, the selected groups could have been widely

364 spread by regional flooding. This is because each group was distributed over a wide area.

365 Further study, however, is needed to assess the relationship between the distribution of a

366 Leptospira group and the area of flooding as well as the possibility of horizontal or vertical

367 transmission, especially among the animal reservoirs.

368 It was found that the PFGE patterns of Groups A and C strains were similar to each

369 other, and the number of different fragments between these strains was only four. DNA

370 Gyrase B gene sequencing results revealed that Group C strains had almost similar sequences

371 (except for one base substitution) with Group A strains. This was a similar phenomenon that

372 we observed in our previous study [17]. Based on these results, strains belonging to these

373 groups may 'probably be related.' According to geographic distribution analysis, the strains of

374 these two groups (A and C) were isolated in different regions (Metro Manila; and Pangasinan

375 and Nueva Ecija, respectively). Although they were different serogroups and from different

376 regions they could, however, be genetically related. 
In our previous study in 2006 [17], serogroup Javanica strains were isolated from

378 rats in Metro Manila and Laguna, which is located on the southwest of Metro Manila, and

379 these strains were categorized as the fourth predominant serogroup (group D). But, in the

380 present study, no Leptospira were isolated belonging to serogroup Javanica. This

381 phenomenon was also observed in the study by Amilasan, et al [16] after the great flood that

382 occurred in the Philippines in 2009. It is unclear why strains belonging to serogroup Javanica

383 were not predominantly isolated anymore. However, there are at least three possibilities.

384 Firstly, strains belonging to this serogroup may still be dominant but we might have missed

385 isolating them due to the limited number of rats that were trapped in our current study.

386 Secondly, Ellinghausen-McCullough-Johnson-Harris (EMJH) medium was possibly essential

387 to isolate strains belonging to serogroup Javanica. Although there was no sufficient evidence,

388 the strains of serovar Javanica tended to favor EMJH medium over Korthof's medium, as we

389 previously reported [17]. In the present study, we only used Korthof's medium to culture the

390 samples. Thirdly, serogroup Javanica could be predominant only transiently. Lau et al. [31]

391 reported the change of predominant serovars of human Leptospira isolates between 2004 and

3922010 in American Samoa. They described that leptospiral serovar epidemiology can be 
393 affected by change in distribution and/or abundance of animal reservoirs, introduction of new

394 serovars through transport of animals or natural migration, or environmental changes.

395 Although all the isolates belong to pathogenic serogroups, not all of the strains

396 caused moribundity in hamsters. Most of the human (72\%) and rat isolates (73\%) were found

397 to be pathogenic to hamsters. It is common knowledge that pathogenic leptospires lose their

398 virulence after being in vitro subcultured many times (more than 10 times). However, in this

399 study, all strains that we used for the pathogenicity test were in vitro passaged less than 10

400 times. The virulence of Leptospira isolates may probably have been lost during transit in the

401 environment or may depend on interaction with unknown host factor/s.

402 In conclusion, we demonstrated a high level of intra-group PFGE pattern similarity

403 and gyrB sequence identity among the Leptospira isolates from humans and rats in Luzon,

404 Philippines. It is suggested that there are mainly three dominant Leptospira groups circulating

405 among rats, and these may have persistently caused leptospirosis in humans over the years

406 particularly in this island. The findings in this study will be very crucial in the development of

407 vaccines, at least in the Philippines, which will greatly contribute to the prevention and

408 control of leptospirosis. 


\section{Acknowledgments}

411

412

We would like to thank Bobby Crisostomo, Hideko Kameyama, Naomi Hidaka,

413 laboratory aides at DMM-CPH-UP Manila, staff of the Philippine Carabao Center in Nueva

414 Ecija, market officers, and vendors for their technical support in this study. We are also deeply

415 indebted to the patients and their guardians for their willingness to cooperate in this study. We

416 would also like to thank the Japan Science and Technology Agency (JST) and Japan

417 International Cooperation Agency (JICA) through their Science and Technology Research

418 Partnership for Sustainable Development (SATREPS) program for giving us the grant to

419 carry out this study. All the opinions and statements in this paper are solely that of the authors

420 and were not, in any way, influenced by the funding agencies nor by the institutions with

421 which the authors are affiliated. 


\section{References}

424

425 [1] Abela-Ridder B, Sikkema R, Hartskeerl RA. Estimating the burden of human leptospirosis.

426 Int J Antimicrob Agents 36 (2010) S5-7.

427

428 [2] Adler B, de la Peña Moctezuma A. Leptospira and leptospirosis. Vet Microbiol 140 (2010)

$429 \quad 287-296$.

430

431 [3] Saravanan R, Rajendran P, Thyagarajan SP, Smythe LD, Norris MA, Symonds ML, et al.

432 Leptospira autumnalis isolated from a human case from Avadi, India, and the serovar's

433 predominance in local rat and bandicoot populations. Ann Trop Med Parasitol 94 (2000)

$434 \quad 503-6$.

435

436 [4] Koizumi N, Muto M, Tanikawa T, Mizutani H, Sohmura Y, Hayashi E, et al. Human

437 leptospirosis cases and the prevalence of rats harbouring Leptospira interrogans in urban

438 areas of Tokyo, Japan. J Med Microbiol 58 (2009) 1227-1230. 
440 [5] Thaipadungpanit J, Wuthiekanun V, Chierakul W, Smythe LD, Petkanchanapong W,

441 Limpaiboon R, et al. A dominant clone of Leptospira interrogans associated with an outbreak

442 of human leptospirosis in Thailand. PLoS Negl Trop Dis 1 (2007) e56.

443

444 [6] World Health Organization and International Leptospirosis Society. Human leptospirosis:

445 Guidance for diagnosis, surveillance and control. World Health Organization and International

446 Leptospirosis Society, Geneva, 2003.

447

448 [7] Galloway RL, Levett PN. Application and validation of PFGE for serovar identification of

449 Leptospira clinical isolates. PLoS Negl Trop Dis 4 (2010) e824.

450

451 [8] Herrmann JL, Bellenger E, Perolat P, Baranton G, Saint Girons I. Pulsed-field gel

452 electrophoresis of NotI digests of leptospiral DNA: a new rapid method of serovar

453 identification. J Clin Microbiol 30 (1992) 1696-1702.

454

455 [9] Romero EC, Blanco RM, Galloway RL. Application of pulsed-field gel electrophoresis for

456 the discrimination of leptospiral isolates in Brazil. Lett Appl Microbiol 48 (2009) 623-627. 
458 [10] Galloway RL, Levett PN. Evaluation of a modified pulsed-field gel electrophoresis

459 approach for the identification of Leptospira serovars. Am J Trop Med Hyg 78 (2008)

$460 \quad 628-632$.

461

462 [11] Kawabata H, Sakakibara S, Imai Y, Masuzawa T, Fujita H, Tsurumi M, et al. First record

463 of Leptospira borgpetersenii isolation in the Amami Islands, Japan. Microbiol Immunol 50

464 (2006) 429-434.

465

466 [12] Masuzawa T, Okamoto Y, Une Y, Takeuchi T, Tsukagoshi K, Koizumi N, et al.

467 Leptospirosis in squirrels imported from United States to Japan. Emerg Infect Dis 12 (2006)

$468 \quad 1153-1155$.

469

470 [13] Slack AT, Symonds ML, Dohnt MF, Smythe LD. Identification of pathogenic Leptospira

471 species by conventional or real-time PCR and sequencing of the DNA gyrase subunit B

472 encoding gene. BMC Microbiol 6 (2006) 95. 
474 [14] Yanagihara Y, Villanueva SY, Yoshida S, Okamoto Y, Masuzawa T. Current status of 475 leptospirosis in Japan and Philippines. Comp Immunol Microbiol Infect Dis 30 (2007)

$476 \quad 399-413$.

477

478 [15] McCurry J. Philippines struggles to recover from typhoons. Lancet. 374 (2009) 1489.

479

480 [16] Amilasan AT, Ujiie M, Suzuki M, Salva E, Belo MC, Koizumi N, et al. Outbreak of

481 leptospirosis after flood, the Philippines, 2009. Emerg Infect Dis 18 (2012) 91-94.

482

483 [17] Villanueva SY, Ezoe H, Baterna RA, Yanagihara Y, Muto M, Koizumi N, et al.

484 Serologic and molecular studies of Leptospira and leptospirosis among rats in the Philippines.

485 Am J Trop Med Hyg 82 (2010) 889-898.

486

487 [18] Villanueva SY, Saito M, Tsutsumi Y, Segawa T, Baterna RA, Chakraborty A, et al. High

488 virulence in hamsters of four dominantly prevailing Leptospira serovars isolated from rats in the Philippines. Microbiology 160 (2014) 418-428. 
491 [19] Aplin KP, Brown PR, Jacob J, Krebs CJ, Singleton GR. Field methods for rodent studies

492 in Asia and the Indo-Pacific. Australian Centre for International Agricultural Research,

493 Canberra, 2003.

494

495 [20] Woo TH, Patel BK, Smythe LD, Symonds ML, Norris MA, Dohnt MF. Identification of

496 pathogenic Leptospira genospecies by continuous monitoring of fluorogenic hybridization

497 probes during rapid-cycle PCR. J Clin Microbiol 35 (1997) 3140-3146.

498

499 [21] Kawabata H, Dancel LA, Villanueva SY, Yanagihara Y, Koizumi N, Watanabe H.

500 flaB-polymerase chain reaction (flaB-PCR) and its restriction fragment length polymorphism

501 (RFLP) analysis are an efficient tool for detection and identification of Leptospira spp.

502 Microbiol Immunol 45 (2001) 491-496.

503

504 [22] Yamamoto S, Harayama S. PCR amplification and direct sequencing of gyrB genes with

505 universal primers and their application to the detection and taxonomic analysis of

506 Pseudomonas putida strains. Appl Environ Microbiol 61 (1995) 1104-1109. 
508 [23] Saito M, Villanueva SY, Chakraborty A, Miyahara S, Segawa T, Asoh T, et al.

509 Comparative analysis of Leptospira strains isolated from environmental soil and water in the

510 Philippines and Japan. Appl Environ Microbiol 79 (2013) 601-609.

511

512 [24] Kmety E, Dikken H. Classification of the species Leptospira interrogans and history of

513 its serovars. University Press, Groningen, 1993.

514

515 [25] Bourhy P, Collet L, Clement S, Huerre M, Ave P, Giry C, et al. Isolation and

516 characterization of new Leptospira genotypes from patients in Mayotte (Indian Ocean). PLoS

517 Negl Trop Dis 4 (2010) e724.

518

519 [26] Masuzawa T, Kumagai M, Shimizu T, Yanagihara Y. Classification of Leptospira

520 interrogans serovar Lai strain 017 by using monoclonal antibodies. J Clin Microbiol 26

$521 \quad$ (1998) 2332-2337.

522

523

524 
527 [27] Rahelinirina S, Léon A, Harstskeerl RA, Sertour N, Ahmed A, Raharimanana C, et al.

528 First isolation and direct evidence for the existence of large small-mammal reservoirs of

529 Leptospira sp. in Madagascar. PLoS One 5 (2010) e14111.

530

531 [28] Bourhy P, Collet L, Lernout T, Zinini F, Hartskeerl RA, van der Linden H, et al. Human

532 Leptospira isolates circulating in Mayotte (Indian Ocean) have unique serological and

533 molecular features. J Clin Microbiol 50 (2012) 307-311.

534

535 [29] Pereira MM, Matsuo MG, Bauab AR, Vasconcelos SA, Moraes ZM, Baranton G, et al. A

536 clonal subpopulation of Leptospira interrogans sensu stricto is the major cause of

537 leptospirosis outbreaks in Brazil. J Clin Microbiol 38 (2000) 450-452.

538

539 [30] Barcellos C, Sabroza PC. Socio-environmental determinants of the leptospirosis outbreak

540 of 1996 in western Rio de Janeiro: a geographical approach. Int J Environ Health Res 10

$541 \quad$ (2000) 301-313. 
543 [31] Lau CL, Skelly C, Smythe LD, Craig SB, Weinstein P. Emergence of new leptospiral

544 serovars in American Samoa - ascertainment or ecological change? BMC Infect Dis 12 (2012)

54519. 


\section{$546 \quad$ Figure Legends}

548 Fig. 1. PFGE patterns of the 40 Leptospira isolates in this study and the seven strains

549 previously isolated in the Philippines using NotI restriction enzyme. Human isolates are

550 highlighted in dark colors and rat isolates in faint color. Closed red circles indicate strains that

551 were isolated before and in 2006. PFGE results of rat and human isolates showed similar

552 PFGE patterns in each group. a, probable serovar based on gyrB sequencing. b, antiserum to

553 the indicated serogroup which showed the highest titer to the isolate.

554

555 Fig. 2. Phylogenetic tree based on the gyrB sequences of the Leptospira strains isolated from

556 humans and rats. All the isolates were determined to belong to L. interrogans. Strains written

557 in bold fonts were the isolates obtained in the current study. Accession numbers of the

558 isolates are written in parentheses. Numbers indicate bootstrap value with 1,000 replicates.

559 Colors represent the PFGE groupings as in Fig. 1. Bar: nucleotide substitution of 0.9\%. Sg:

560 serogroup. Sg: serogroup. Sv: serovar. Str: strain. 
562 Fig. 3. Geographic distributions of culture-positive patients and rats. The addresses of the

563 patients and the sample collection sites of the rats were obtained and plotted in the map using

564 ArcGIS. Colors of the symbols represent the PFGE groupings similar to Fig. 1.

565

566 
567 Table 1. Distribution of Leptospira isolates from humans and rats according to sample

568 collection sites.

\begin{tabular}{llll}
\hline & No. of isolates from & \\
Sample collection sites & & Total \\
& humans & Rats & \\
\hline
\end{tabular}

\section{A. Luzon}

(1) National Capital Region

8

9

17

(Metro Manila)

(2) Regions I/Ilocos Region

(Pangasinan) and

10

2

12

III/Central Luzon (Nueva

Ecija)

(3) Region

IV-A/CALABARZON

6

0

6

(Laguna and Cavite)

(4) Region V/Bicol Region

$\begin{array}{lll}1 & 0 & 1\end{array}$

(Camarines Sur)

\section{B. Visayas}


Region VI/Western Visayas

$\begin{array}{lll}0 & 2 & 2\end{array}$

(Iloilo)

C. Unknown address

2

0

2

Total

27

13

40

569 
570 Table 2. Serotyping results of Leptospira isolates from humans and rats.

\begin{tabular}{|c|c|c|c|}
\hline \multirow{2}{*}{ Serogroup } & \multicolumn{2}{|c|}{ No. of isolates from } & \multirow{2}{*}{ Total } \\
\hline & humans & rats & \\
\hline Pyrogenes & 13 & 8 & 21 \\
\hline Bataviae & 7 & 1 & 8 \\
\hline Grippotyphosa & 6 & 2 & 8 \\
\hline Canicola & 1 & 0 & 1 \\
\hline Icterohaemorrhagiae & 0 & 2 & 2 \\
\hline Total & 27 & 13 & 40 \\
\hline
\end{tabular}

571 
Figures

\section{Figure 1}

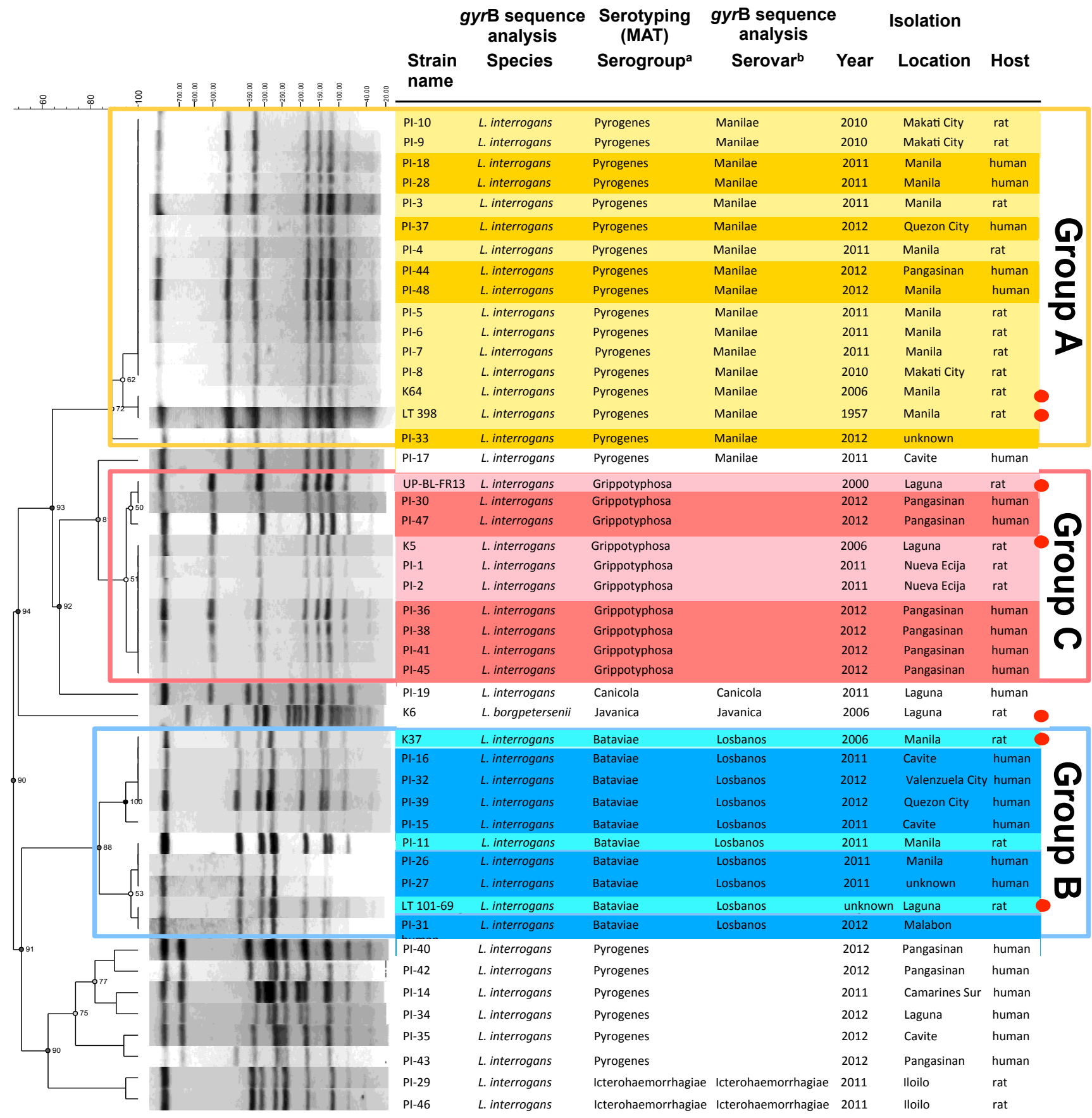

Previous Isolates 


\section{Figure 2}

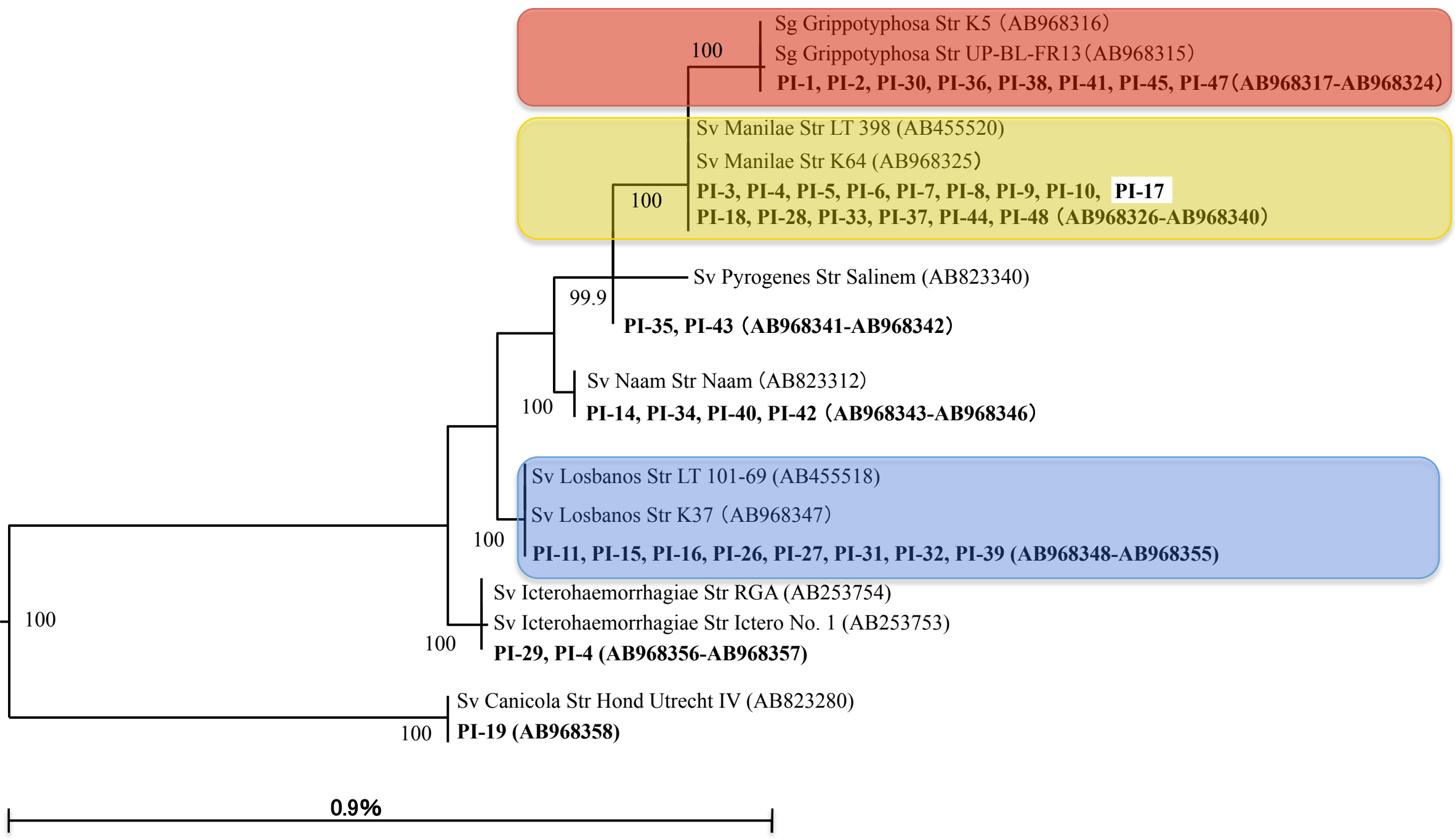




\section{Figure 3}
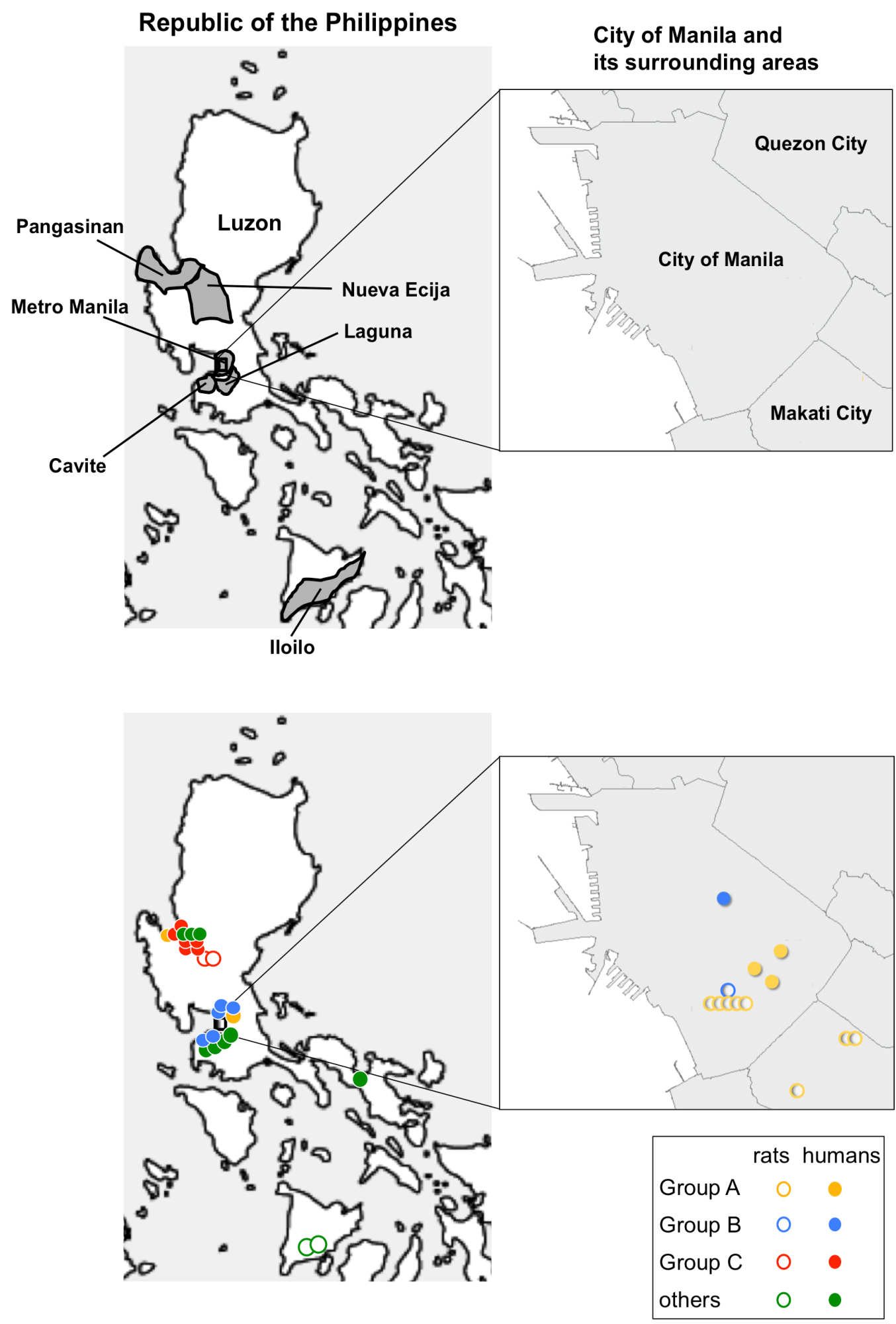